\title{
Detection and Classification of Landmines using AR Modeling of GPR Data
}

\author{
Daniela Deiana, Laura Anitori \\ TNO Defence, Security and Safety \\ The Hague, The Netherlands \\ daniela.deiana@tno.nl
}

\begin{abstract}
In this paper we present some results on detection and classification of low metal content anti personnel (AP) landmines using a modified version of the Auto Regressive (AR) modeling algorithm presented in $[1,2]$. A statistical distance is computed between the AR coefficients of the measured GPR time signal and the AR coefficients of a reference database (containing the AR models of the mines of interest) and a detection is declared if this distance is below a given threshold.
\end{abstract}

\section{Keywords- AR Modeling; Landmines; GPR}

\section{INTRODUCTION}

AR modeling algorithms have been used in GPR, amongst others, for clutter reduction [3,4] and target detection [5]. In this paper we present some developments on detection and classification of low metal content antipersonnel landmines using the AR modeling algorithm presented in [1,2]. This algorithm is used for generating an AR model (i.e. AR coefficients) for each A-scan [6] of a B-scan obtained from a Ground Penetrating Radar (GPR).

The AR modeling algorithm computes a similarity measure between the AR coefficients of a reference database and the measured GPR trace. A GPR time signal varies with the electromagnetic properties of the terrain, with the depth and the orientation of the target. This means that the same landmine will be modeled with different poles depending on the circumstances. With this research we want to study the sensitivity of the AR model, i.e. how different the AR model coefficients will be if we measure the same mine in a different soil type, or in the same soil but with a different moisture, or when the mine is buried at a different depth, or the mine is tilted with respect to the database values. For this purpose we have simulated a number of mines, with both very low metal content (SIM6, SIM12, SIM20) and with a metal case (SIM20Al) using the GPRmax software tool [7] and we varied the burial depths and the mine orientations.

The paper is organized as follows. Section II describes the process of creating a dataset of simulant mines (SIMs) with the software GPRMax and the relative Bscans. In section III the AR model for mine detection and classification is described. The results for the SIMs are shown in section IV, while section $\mathrm{V}$ presents the preliminary results obtained from real data. Discussion of the results and conclusions are the subject of section VI.

\section{GPRMAX}

GPRMax is an electromagnetic wave simulator for GPR modelling in $2 \mathrm{D}$ and in $3 \mathrm{D}$, based on the Finite-Difference Time-Domain (FDTD) numerical method.

For the purposes of this research the two-dimensional GPRMax simulator has been used. The modeled GPR is a bistatic pulsed radar with central frequency at $900 \mathrm{MHz}$. The transmitter emits a Ricker wavelet, with a time window of 10 ns. The antenna has been placed $5 \mathrm{~cm}$ above the ground. The radar scans a line 1 meter long with a step of $1 \mathrm{~cm}$.

The targets are three low metal content SIMs of diameter 6 $\mathrm{cm}$ (SIM6), $12 \mathrm{~cm}$ (SIM12) and $20 \mathrm{~cm}$ (SIM20) respectively, and an aluminium SIM with a diameter of $20 \mathrm{~cm}$ (SIM20Al). The plastic mines contain a small aluminium tube with a length of $1.27 \mathrm{~cm}$ and a diameter of $0.475 \mathrm{~cm}$.

The electromagnetic properties of the SIMs are those described by the ITOP guidelines [8]. The properties of the SIMs components (the cover (abs), the explosive (RTV) and the aluminium tube), as well as those of the sand are reported in table I.

TABLE I. Electromagnetic Properties of the Materials

\begin{tabular}{|l|l|l|}
\hline Material & $\begin{array}{l}\text { Relative } \\
\text { Permittivity }\end{array}$ & Conductivity[S/m] \\
\hline Dry Sand & 5.0 & 0.001 \\
\hline Wet Sand & 20.0 & 0.01 \\
\hline Aluminium & 8.6 & $2.3 \cdot 10^{7}$ \\
\hline RTV & 3.1 & 0.0 \\
\hline abs & 2.81 & 0.0 \\
\hline
\end{tabular}

The SIM6 and SIM12 are placed at a shallow depth of 2, 4 and $5 \mathrm{~cm}$, and at each depth a simulation is performed for tilt angles of $0^{\circ}, 5^{\circ}$ and $20^{\circ}$ respectively. SIM20 and SIM20Al are placed at a depth of $15 \mathrm{~cm}$ and tilted of $0^{\circ}, 5^{\circ}$ and $20^{\circ}$. Simulations were carried out in both dry and wet sand. Figure 3 (top) shows a representative geometry of the simulations. 


\section{AR-MODELING}

The detection algorithm used in this work is based on autoregressive (AR) modeling of time series. This technique has already been successfully used in speech processing and a Matlab package (ARFIT) [9] is available for AR model parameters estimation.

The autoregressive model of order $p$ of a time series $y_{n}$ can be written as

$$
y_{n}^{m d}=x_{n}-\sum_{i=1}^{p} a_{i} \cdot y_{n-i},
$$

where $a_{i}(i=1, \ldots, \mathrm{p})$ are the autoregressive coefficients, obtained by minimizing the mean-squared difference between the modeled time series $y_{n}^{m d}$ and the observed one $y_{n} \cdot x_{n}$ is the input signal.

The comparison of two Ascan patterns is performed using the Itakura-Saito distortion measurement [10]. It is a likelihood distortion algorithm that calculates the statistical distance between two AR models. Given the residual mean square error

$$
E^{(p)}=\sum_{n=0}^{N-1+p} e_{n}^{2}=\sum_{n=0}^{N-1-p}\left[-\sum_{i=0}^{p} a_{i} x_{n-i}\right]^{2}=a^{t} \cdot \underline{\underline{R}} \cdot a
$$

where $\underline{\underline{R}}$ is the residual autocorrelation matrix, the ItakuraSaito (IS) statistical distance is calculated as:

$$
D=\ln \left(\frac{E_{\text {meas }}^{(p)}}{E_{r e f}^{(p)}}\right)=\ln \left(\frac{a_{m}^{t} R a_{m}}{a_{r}^{t} R a_{r}}\right),
$$

where $a_{m}$ is the vector of the AR coefficients of the measured Ascan, while $a_{r}$ is the vector of the AR coefficients of the database. Two AR models are considered similar when the IS distance is small. When they are equal, the distance is equal to zero.

In the Matlab package ARFIT a stepwise least squares algorithm is used for estimating the coefficients of the AR model of a given time serie.

The determination of the order of the model is a complex and very important step in AR modeling. A high order means a better representation of the signal, but also large computation time of the coefficients. On the other hand, a too small order does not correctly represent the signal. In the area of speech recognition the optimum order is calculated with an information based criterion (i.e. Akaike Information Criterion or Schwarz Bayesian Criterion). For GPR time signals conventional criteria seem not to estimate the order correctly and therefore the order has been determined with an iterative IS distance based method. Given a predetermined range of orders, the optimal order of the AR model of a target is that one for which the IS distance of the target model with the background model is maximum.
This selection of the optimal order of the AR model of each Ascan is explained in the following steps:

1. The Bscan data is pre-processed in order to remove direct coupling and ground reflection;

2. An Ascan representative for the background is obtained by averaging the Ascans of the Bscan with an energy threshold (i.e. Ascans containing the target are not used in the average);

3. An AR model of the estimated mean background Ascan is calculated;

4. The Itakura-Saito distance between the AR models of all Ascans in the Bscan and the AR model of the estimated mean background is computed for a specific range of orders (i.e. from order 5 to order 20). When a Ascan relative to the target is compared to the Ascan of the background, this distance should be maximum. For example, given the Bscan of figure 1 (top) and selected an initial order for the AR model (i.e. 5), each Ascan is compared with the background Ascan and the distance is calculated. The procedure is repeated until the maximum predefined order is reached (i.e. 20). All the distances relative to the orders are plotted in figure 1 (bottom). The optimal order corresponds thus to the maximum distance.

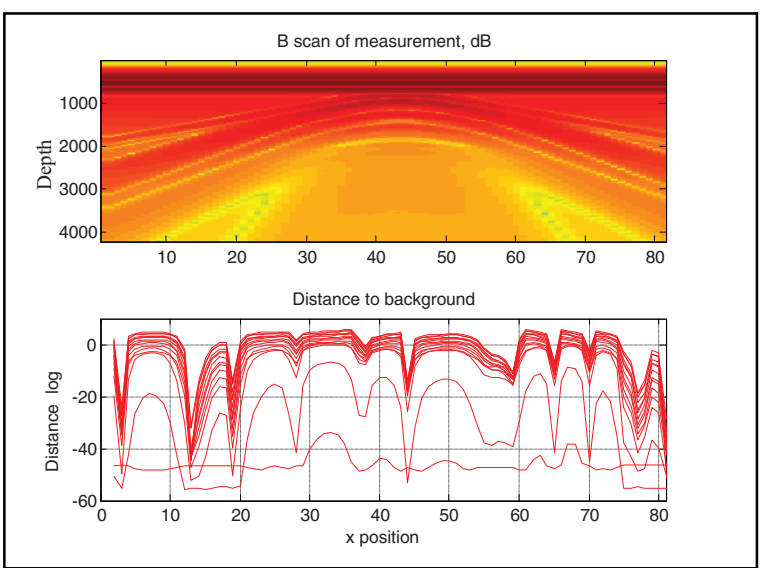

Figure 1 Bscan of a target and distances of the target with the background. Each red curve in the bottom plot corresponds to an order.

\section{Target Detection}

The AR model of each Ascan of a Bscan is compared to the database AR models. The minima of the IS distances correspond to targets. The algorithm automatically classifies the targets.

\section{RESULTS OF THE SIMULATIONS}

The GPRMax tool has been used to simulate Bscans of the reference SIMs used to build up the database and Bscans of the SIMs at various positions and orientations. The dataset of the reference SIMs is given in table II. 
TABLE II. DATABASE SIMS

\begin{tabular}{|c|c|c|}
\hline SIM Type & Depth [cm] & Tilt angle [deg] \\
\hline SIM6 & 4 & 0 \\
\hline SIM12 & 5 & 0 \\
\hline SIM20 & 15 & 0 \\
\hline SIM20A1 & 15 & 0 \\
\hline
\end{tabular}

For each SIM one Ascan AR model is taken. The representative Ascan is the one containing the apex of the hyperbola.

Per each simulated Bscan, an AR model is calculated for every Ascan and it is compared with the database AR models. Figure 2 shows the distribution of the poles of three simulations of the SIM6 at different depths and tilt angles and buried in dry and wet sand. Some of the poles have significantly changed their positions and it does not seem possible to determine by visual inspection whether the poles are relative to the same SIM or not. We are trying to establish whether the AR algorithm is able to correctly classify the SIM6 even if the poles have changed respect to the database model.

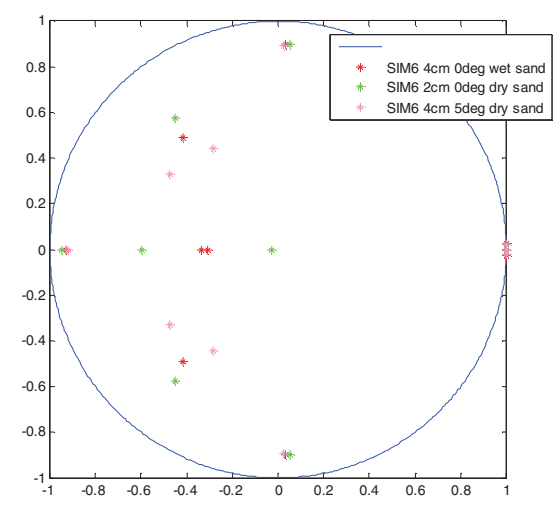

Figure 2 Poles of the AR model of three Ascans of the SIM6

Figure 3, 4 and 5 show the results of the classification of a SIM6 at different depths and orientations by using the database of table II. The top figure shows the geometry. The simulated Bscan is plotted at the middle and the distances are plotted at the bottom of the figure. The SIM6 is always correctly classified. It is interesting to notice that when the SIM6 is tilted $20^{\circ}$, the algorithm shows a relative minimum of $-12 \mathrm{~dB}$ for the SIM12 at point 38 in the $\mathrm{x}$ axis, only $2 \mathrm{~dB}$ less than for the SIM6. The two mines are made of the same material, they differ only in their size. A SIM6 tilted $20^{\circ}$ has a thicker cross section, thus its Ascan is more similar to that one of the SIM12.

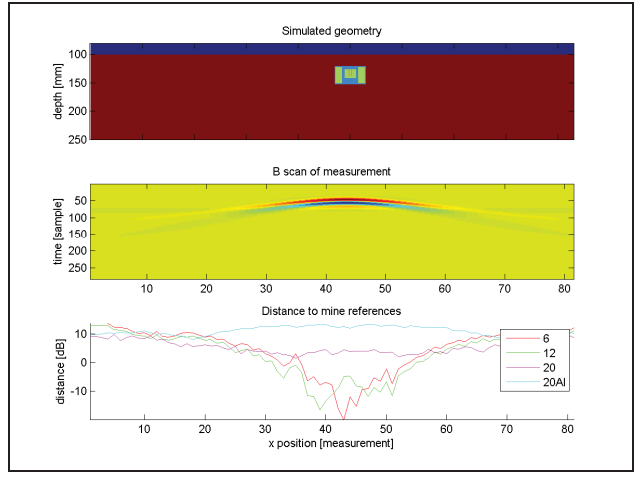

Figure 3 SIM6 buried at $2 \mathrm{~cm}$, tilt angle $0^{\circ}$

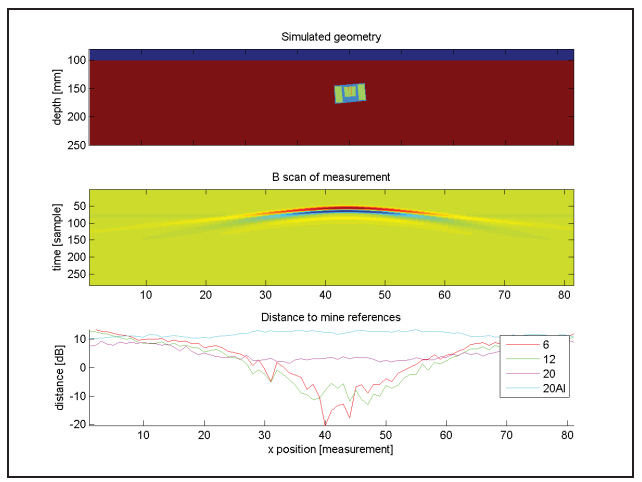

Figure 4 SIM6 buried at $4 \mathrm{~cm}$, tilt angle $5^{\circ}$

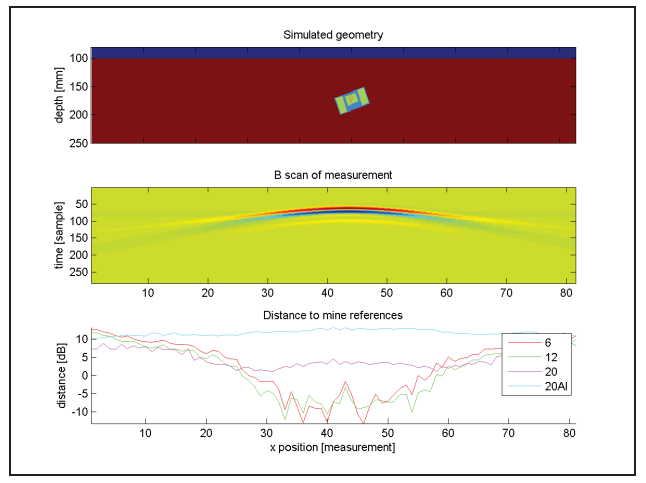

Figure 5 SIM6 buried at $5 \mathrm{~cm}$, tilt angle $20^{\circ}$

Table III summarizes the results of all the simulations in dry sand. All the simulated Bscans are compared to the reference SIMs, denoted with the asterisk. Next to each classification the minimum distance in log scale is shown. The leftmost classification result is the best one (smallest minimum distance). The asterisk denotes the reference SIM. All the SIM6 and SIM12 are correctly detected and classified, while the SIM20 and the SIM20Al are classified correctly only once. So we conclude that the performance of the algorithm is good even with a limited database. 
TABLE III. ClassificATION OF SIMS

\begin{tabular}{|l|l|}
\hline SIM Type & Target Classification \\
\hline SIM6 2cm 0deg & SIM6 (-20), SIM12 (-15) \\
SIM6 2cm 5deg & SIM6 (-15), SIM12 (-15) equal distance \\
SIM6 2cm 20deg & SIM6 (-15), SIM12 (-13) \\
SIM6 4cm 0deg * & \\
SIM6 4cm 5deg & SIM6 (-20), SIM12 (-12) \\
SIM6 4cm 20deg & SIM6 (-15) \\
SIM6 5cm 0deg & SIM6 (-15), SIM12 (-11) \\
SIM6 5cm 5deg & SIM6 (-15) \\
SIM6 5cm 20deg & SIM6 (-14), SIM12 (-12) \\
\hline SIM12 2cm 0deg & SIM12 (-12), SIM6 (-11) \\
SIM12 2cm 5deg & SIM12 (-15), SIM6 (-12) \\
SIM12 2cm 20deg & SIM12 (-15) \\
SIM12 4cm 0deg & SIM12 (-15) \\
SIM12 5cm 0deg * & \\
\hline SIM20 15cm 0deg & \\
SIM20 15cm 5deg & SIM20Al (-20), SIM20 (-15) \\
SIM20 15cm 20deg & SIM20 (-15), SIM20Al (-12) \\
\hline SIM20Al 15cm 0deg * & \\
SIM20Al 15cm 5deg & SIM20 (-15),SIM6 (-13), SIM20A1 (-12) \\
SIM20A1 15cm 20deg & SIM20Al (-21), SIM20 (-18) \\
\hline
\end{tabular}

\section{RESUlTS OF THE REAL DATA}

The measurements have been done at the land-mine test facility at TNO-FEL. The radar used is the PulseEKKO 900 $\mathrm{MHz}$ GPR, placed $5 \mathrm{~cm}$ above the ground. Firstly the database has to be created. Four reference SIMs have been used. SIM9 and SIM12 have been buried at a depth of $5 \mathrm{~cm}$, while the depth of SIM20 and SIM25 was $7 \mathrm{~cm}$. All SIMs were not tilted and the tests were done in dry sand. Again, the representative Ascan of each SIM is that one containing the apex of the hyperbola. The AR model of each Ascan has been recorded in the database.

Once the database is complete, the measurements can take place. 5 SIMs, a ferromagnetic rock and a "Hero" can have been buried in dry sand along 3 lanes. The revealed SIMs are shown in figure 6.

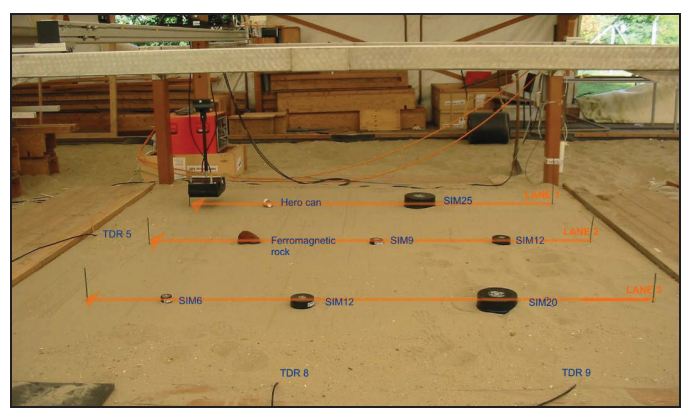

Figure 6 Measurement setup

The preliminary results are shown in figures 7,8 and 9. At the top of each figures the Bscan is plotted (after background removal and alignment), while at the bottom are shown the IS distances of the Ascans with the database records.

The algorithm is not able to classify the objects correctly: the relative minima are only at $-4 \mathrm{~dB}$ from the average value.

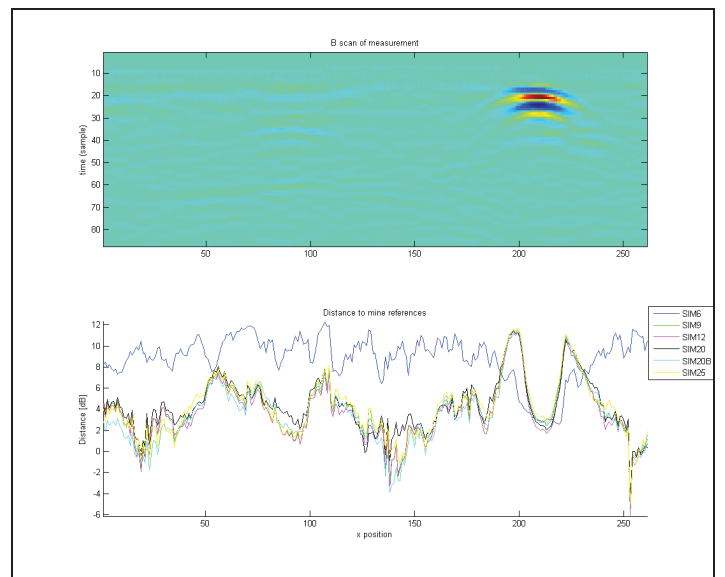

Figure 7 (top) Bscan of Lane 1: SIM25 $(x=91 \mathrm{~cm})$, Hero can $(x=210 \mathrm{~cm})$. (bottom) IS distances

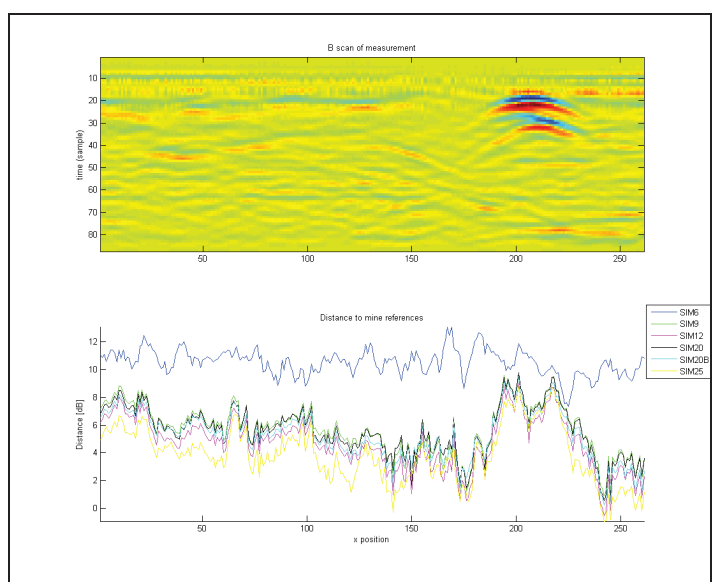

Figure 8 (top) Bscan of Lane 2: SIM12 ( $\mathrm{x}=44 \mathrm{~cm}), \operatorname{SIM} 9(\mathrm{x}=124 \mathrm{~cm})$, ferromagnetic $\operatorname{rock}(\mathrm{x}=205 \mathrm{~cm})$. (bottom) IS distances.

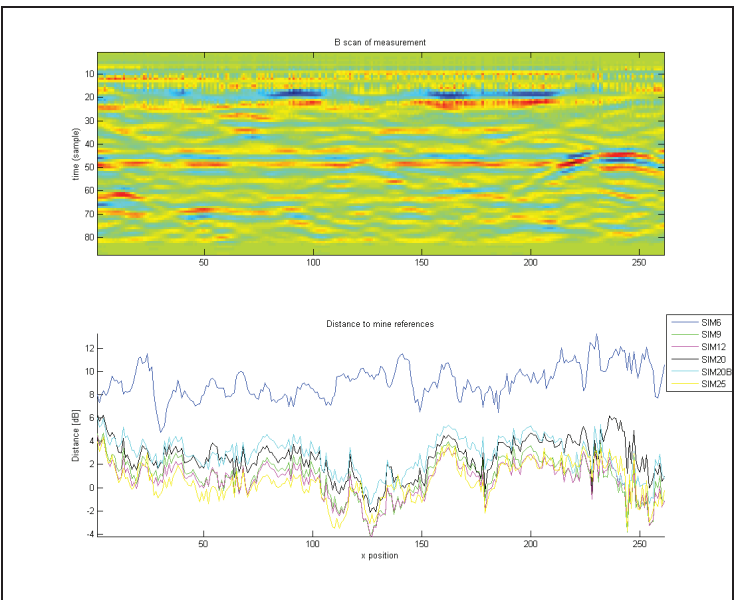

Figure 9 (top) Bscan of Lane 3: SIM20 ( $x=64 \mathrm{~cm}), \operatorname{SIM} 12(\mathrm{x}=160 \mathrm{~cm})$, SIM6 ( $\mathrm{x}=190 \mathrm{~cm})$. (bottom) IS distances.

Please note that the database was built taking only one Ascan per each reference SIM, which seemed good enough for classification when used with simulated data. An explanation could be that a homogeneous terrain was used and no losses were considered. For real data, the results alone are not 
sufficient to judge the performance of the algorithm. It is necessary to make more simulations where the losses and inhomogeneities are taken into account and verify whether it is necessary to increase the number of records in the database, as explained in the next section.

\section{DISCUSSION AND CONCLUSIONS}

In this paper we proposed a modified version of the AR model algorithm for landmine classification. The algorithm is based on comparison of measured data with the database and we have studied how the classification is affected by mismatch between the training set and the measured data, i.e. the same mine has a different depth in the database and in the measured data. We tried to establish this sensitivity with both simulated and real data. Firstly the algorithm has been applied to Bscans obtained with the software tool GPRMax. For each Ascan an AR model is computed and the classification is been performed by minimizing the Itakura-Saito statistical distance with the Ascans in the database. With simulated data the algorithm performs well and the results are satisfactory. In $80 \%$ of the cases the classification was correct.

Preliminary results on real data show that the algorithm is very sensitive to discrepancies between the database and the measured data. Nevertheless the database for the real data contained only one Ascan per SIM.

The difference between the simulation and the measurements could be explained by the fact that in the simulated data the losses and the inhomogeneities of the medium have not been taken into account. The next step would be to include these variables in the simulations and check whether the results are still good or if it is necessary to add records into the database. On the other hand, a high number of records can bring to misclassification.

An analysis of the behavior of the AR models of the database should be done, in order to understand their variation with respect to different positions, orientations and depths of the SIMs and with different soil properties.

Moreover, although we have collected two dimensional data, we have only used the one dimensional time (depth) signal for matching the measured data to the database. A second improvement to the algorithm could be building a database with all the consecutive Ascans containing the signature of the SIM. The statistical distance would then be calculated for an Ascan-block instead of for a single Ascan.

\section{FUTURE WORK}

Next to the AR model, we are studying other techniques for the classification of landmines. In particular we are working with 3D GPR data. Migration techniques and geometrical and statistical feature extraction will be used for target detection and classification.

\section{REFERENCES}

[1] J. B. Rhebergen, H. A. Lensen, R. van Wijk, J.M.H. Hendrickx, L. van Dam, and B.Borchers, "Prediction of soil effects on GPR signatures", Defence and Security Symposium, Proc. SPIE, Vol. 5415, 705(2004).

[2] J. B. Rhebergen, R. van Wijk, "Model based detection and identification of land-mine signatures in GPR-data", $10^{\text {th }}$ International Conference on Ground Penetrating Radar, 21-24 June, 2004, Delft, The Netherlands

[3] V. Venkatasubramanian, H. Leu7ng, B. Moorman, "An Interacting Multiple-Model-Based Abrupt Change Detector for Ground-Penetrating Radar", IEEE Geoscience and Remote Sensing Letters, Vol. 4, No. 4, October 2007

[4] V. L. Kempen and H. Sahli, "Signal Processing Techniques for Clutter Parameters Estimation and Clutter Removal in GPR Data for Landmine Detection", Statistical Signal Processing, 2001. Proceedings of the $11^{\text {th }}$ IEEE Signal Processing Workshop, 158-161, 2001

[5] J. Kositsky, P. Milanfar, "A Forward-Looking High-Resolution GPR system"

[6] D.J. Daniels, "Ground Penetrating Radar", $2^{\text {nd }}$ Edition, The Institution of Electrical Engineers, 2004

[7] http://www.gprmax.org

[8] http://www.itep.ws/standards/std.html

[9] http://www.gps.caltech.edu/ tapio/arfit/

[10] L. Rabiner, B.H. Juang, "Fundamentals of speech recognition," Prentice Hall, New Jersey, 1993. 